\title{
EDUCAÇÃO EM CONTEXTO DE CULTURA DIGITAL: POTÊNCIAS PEDAGÓGICAS E POSSIBILIDADES DE VISIBILIDADE PARA O CONHECIMENTO CIENTÍFICO ESCOLAR
}

\author{
Education in the digital culture context: pedagogical potency and visibility possibilities \\ for school scientific knowledge
}

\author{
Luciana Domingues Ramos* \\ Cíntia Inês Boll ${ }^{* *}$
}

\begin{abstract}
Resumo: O artigo tem por objetivo apresentar considerações relacionadas à cultura digital, seus desafios e possibilidades na Educação. O recorte principal desta produção é o potencial pedagógico de um trabalho apoiado pelas mídias, pelas linguagens e convergências digitais em direção à construção do conhecimento na perspectiva ética-estética dessa relação. $\mathrm{O}$ tensionamento é provocado com contribuições de autores como Pierre Lévy, Massimo Canevacci, Henry Jenkins, Martha Marandino, Myriam Krasilchik, entre outros estudiosos desse mundo da educação, comunicação e cultura digital. $O$ trabalho conclui que o contexto amplificado e convergente da cultura digital pode ser visto sob uma perspectiva que o entenda como um aliado da escola, não somente no acesso à informação e ao conhecimento, mas um mobilizador de propostas participativas de educação, em oposição a uma educação bancária, que ressignifica as relações, visibiliza as construções da escola, o conhecimento científico escolar e viabiliza a formação de um sujeito autor, colaborador, comunicador, ético e cidadão também em contexto digital.
\end{abstract}

Palavras-chave: Educação. Ensino de Ciências. Cultura da convergência.

\begin{abstract}
This paper aims to present considerations related to the digital culture, its challenges and possibilities for Education. The focus of this study is the pedagogical potential of work supported by the media, by the digital convergences and languages towards the construction of knowledge under the ethical-aesthetical perspective of this relationship. Tension is built up by the contributions of authors such as Pierre Lévy, Massimo Canevacci, Henry Jenkins, Martha Marandino, Myriam Krasilchik, among other scholars of the world of education, communication and digital culture. This paper concludes that the amplified and convergent context of the digital culture may be seen under the perspective that understands it as an ally of the school, not only regarding access to information and knowledge, but also as a way of mobilizing participatory proposals in education, as opposed to a banking education, which redefines the relations, makes the constructions and the scientific knowledge in the school
\end{abstract}

\footnotetext{
* Mestranda do PPG Educação em Ciências: Química da Vida e Saúde (UFRGS). Especialista em Mídias na Educação (UFRGS). Graduada em Ciência Biológicas (Unisinos). Professora das Redes Estadual do RS e Municipal de São Leopoldo. Orcid id: orcid.org/0000-0001-6270-1978 E-mail: ludomingues_sl@yahoo.com.br

** Pedagoga, Mestre e Doutora em Educação pela Faculdade de Educação da Universidade Federal do Rio Grande do Sul-UFRGS, na linha de Pesquisa de Educação, Arte, Linguagem e Tecnologia. Diretora do Departamento de Cursos e Políticas da Graduação- DCPGRAD-UFRGS. Líder do Grupo de Pesquisa CNPq, LELIC - Laboratório de Estudos em Linguagem Interação Cognição/Criação, na linha de Pesquisa PROVIA: Comunidades Virtuais de Aprendizagem. Orcid id: orcid.org/0000-0003-1089-3271. E-mail: cintiaboll@ gmail.com
} 
visible and enables the development of a subject that is an author, collaborator, communicator, an ethical citizenship also in the digital context.

Keywords: Education. Teaching Sciences. Convergence Culture.

\section{Introdução}

As tecnologias de comunicação configuram-se elementos determinantes nos processos culturais e sociais. A aprendizagem assistida pelo computador ou por qualquer outro dispositivo móvel ou tecnologia emergente se consolida como um importante suporte para os processos de construção de cada um.

Atuando neste contexto, a Educação vislumbra, entre as diversas possibilidades existentes, não reduzir o uso da tecnologia a somente disponibilizá-la sem que haja construções, discussões e reflexões a respeito. Outra questão que se apresenta é que as possibilidades atuais que a tecnologia oferece deixam em evidência os benefícios da colaboração e do compartilhamento, promovendo a autoria e o protagonismo de todos os envolvidos neste processo de construção da cidadania digital, na qual o conhecimento aberto, ampliado e democraticamente colocado ao alcance de todos possa ser um dos grandes instrumentos de formação e afirmação dos sujeitos.

Este artigo trata do aluno sob uma perspectiva de sujeito ativo na sua aprendizagem e do professor como um agente no papel de um orientador de caminhos, problematizador, estrategista do processo de aprendizagem, um mediador do diálogo que se estabelece entre o sujeito e o mundo. Perspectiva alinhada com Moran (2013), que ao tensionar a discussão acerca de um processo de aprendizagem no ensino formal, apresenta o que pode experienciar um sujeito inserido em um processo de aprendizagem ativa e compartilhada:

\footnotetext{
É na síntese dinâmica da aprendizagem personalizada e colaborativa que desenvolvemos todo o nosso potencial como pessoas e como grupos sociais, ao enriquecer-nos mutuamente com as múltiplas interfaces do diálogo dentro de cada um, alimentando e alimentados pelos diálogos com os diversos grupos nos quais participamos, com a intensa troca de ideias, sentimentos e competências em múltiplos desafios que a vida nos oferece. (MORAN, 2013, p.5)
}

Fiori (apud FREIRE, 1987, p. 7) afirma que "com a palavra, o homem se faz homem. Ao dizer sua palavra, pois, o homem assume conscientemente sua essencial condição humana." Não surgem, então, no contexto tecnológico atual, diferentes possibilidades de o sujeito "dizer sua palavra", tirando-o da posição de mero espectador para um sujeito com acesso à informação e com poder de opinião? Ao mesmo tempo, esta ferramenta aberta a infinitas possibilidades torna possível a imersão em uma teia perigosa de desinformação e inverdades. O "está na internet" deve ser questionado, levando o sujeito a buscar a confiabilidade da fonte e do interlocutor. O processo em questão é o de construção da cidadania digital, no qual a informação colocada ao alcance de todos vislumbra-se uma aliada dos processos de construção. Nesse sentido, a possibilidade de participação leva a outro patamar a condição do sujeito, o que já era apontado como determinante por Paulo Freire:

Se na imersão era puramente espectador do processo, na emersão descruza os braços e renuncia à expectação e exige a ingerência. Já não se satisfaz em assistir. Quer participar. A sua participação, que implica numa tomada de consciência apenas e não 
ainda numa conscientização — desenvolvimento da tomada de consciência — ameaça às elites detentoras de privilégios. (FREIRE,1967, p.54)

A internet configura-se recurso importante para a Educação tanto na possibilidade de alicerçar as ações em pilares como comunicação e aprendizagem colaborativa, como no compartilhamento do conhecimento construído. Lévy (1999) propõe esta relação da humanidade com o saber, agora inserido na cibercultura. Os conceitos de cibercultura e ciberespaço são apresentados na obra de Lévy e, a partir deles, se estabelecem nossas reflexões:

O termo especifica não apenas a infraestrutura material da comunicação digital, mas também o universo oceânico de informação que ela abriga, assim como os seres humanos que navegam e alimentam esse universo. Quanto ao neologismo 'cibercultura', especifica aqui o conjunto de técnicas (materiais e intelectuais), de práticas, de atitudes, de modos de pensamento e de valores que se desenvolvem juntamente com o crescimento do ciberespaço (LÉVY, 1999, p. 17).

Neste contexto, o processo de aprendizagem demanda um professor que abandone o papel centralizador do conhecimento para assumir um papel de articulador de uma inteligência que é coletiva:

\begin{abstract}
Devemos construir novos modelos do espaço dos conhecimentos. No lugar de representação em escalas lineares e paralelas, em pirâmides estruturadas em 'níveis', organizadas pela noção de pré-requisitos e convergindo para saberes 'superiores', a partir de agora devemos preferir a imagem em espaços de conhecimentos emergentes, abertos, contínuos, em fluxo, não lineares, se reorganizando de acordo com os objetivos ou os contextos, nos quais cada um ocupa posição singular e evolutiva" (LÉVY, 1999, p. 158).
\end{abstract}

Percebe-se com isso a importância da escola neste papel de formação e a necessidade de um professor proficiente nas múltiplas linguagens da cultura digital para atender esta demanda. Boll e Kreutz (2009) apontam sobre a potência pedagógica de atribuir outros conceitos ao espaço escolar, voltados para a cultura digital, estabelecendo formas de práticas de gestão do conhecimento e pensando sua dinâmica nos múltiplos tempos e espaços educacionais.

\begin{abstract}
A Cultura Digital é um campo vasto e potente, pois pode estar articulada com qualquer outro campo além das tecnologias, como por exemplo a arte, a educação, a filosofia, a sociologia, etc. Nesta perspectiva a Cultura Digital, assim como uma proposta de educação integral, maximiza todos os campos dos saberes dispostos, tanto dentro quanto fora do espaço escolar justamente por encontrar-se em um lugar que não pode fechar-se para o seu entorno, que o está desafiando a novos jeitos de aprender (BOLL; KREUTZ, 2009, p11).
\end{abstract}

É inegável a presença das diversas tecnologias digitais, coexistindo e referenciando o modelo de sociedade em que nos encontramos. A cultura da convergência digital (JENKINS, 2009) apresenta outras formas de arranjo do pensamento, de interação e comunicação e, portanto, de aprendizagem: 
[...] a convergência representa uma mudança de paradigma - um deslocamento de conteúdo de mídia específico em direção a um conteúdo que flui por vários canais, em direção a uma elevada interdependência de sistemas de comunicação, em direção a múltiplos modos de acesso a conteúdos de mídia e em direção a relações cada vez mais complexas entre a mídia corporativa, de cima para baixo, e a cultura participativa, de baixo para cima. (JENKINS, 2009, pg. 325)

Estabelecido este novo patamar de relação com a informação, a escola coloca-se como um dos espaços institucionais de formação deste sujeito aluno, usuário, comunicador, autor, ator, espectador, cidadão digital.

\section{Cultura digital e a convergência das mídias: potência pedagógica}

A cultura digital possibilita o estabelecimento de uma dinâmica social de acesso e compartilhamento de informações. Nesse contexto, Fraga (2012) fortalece o entendimento das relações que se estabelecem entre tecnologia, a cultura e humanidade afirmando que os fluxos que se estabelecem entre tecnologia e humanidade raramente são unidirecionais. Ao apresentar que as atividades não se podem separar mais das tecnologias que as constituem, a autora aponta que não é tão simples observar um distanciamento destes elementos. O cenário é de transgressão de limites, temporalidades e territórios, o que exige uma "reconsideração nova de tecnologia, não mais ligada ao poder-fazer, mas ao poder-saber" (FRAGA, 2012, p.30). Este entendimento acerca de tecnologia, humanidade e cultura, nestes termos apresentados pelas autoras, fortalece a compreensão do contexto da cultura digital e de convergência de mídias.

Jenkins et al. (2006 - tradução nossa) abordam a cultura da convergência considerando esferas culturais e sociais - incluindo a Educação - e reafirmando que uma evolução tecnológica altera a forma com que as pessoas vivem, se informam e se comunicam. Nesta discussão, abordando o contexto em que múltiplas linguagens emergem e confluem em diversas bases e plataformas, o autor elenca e aborda uma série de competências culturais necessárias em um cenário de convergência midiática (quadro 1), indicando que o atendimento a essas demandas possibilita aos sujeitos ampliar sua capacidade de percepção, comunicação, interpretação e relação com a informação.

Quadro 1 - competências culturais de habilidades sociais que os jovens precisam no novo cenário da mídia

\begin{tabular}{|l|l|}
\hline \multicolumn{1}{|c|}{$\begin{array}{c}\text { COMPETÊNCIA } \\
\text { CULTURAL }\end{array}$} & \multicolumn{1}{c|}{ CAPACIDADE/HABILIDADE } \\
\hline Brincar & $\begin{array}{l}\text { Experimentar o meio como forma de resolução de } \\
\text { problemas }\end{array}$ \\
\hline Performance & $\begin{array}{l}\text { Adotar identidades alternativas para fins de } \\
\text { improvisação e descoberta }\end{array}$ \\
\hline Simulação & $\begin{array}{l}\text { Interpretar e construir modelos dinâmicos do mundo } \\
\text { real }\end{array}$ \\
\hline Apropriação & $\begin{array}{l}\text { Amostrar e remixar conteúdo de mídia de maneira } \\
\text { significativa }\end{array}$ \\
\hline Multitarefa & $\begin{array}{l}\text { Digitalizar o ambiente e mudar o foco conforme } \\
\text { necessário para destacar detalhes. }\end{array}$ \\
\hline Cognição distribuída & $\begin{array}{l}\text { Interagir de forma significativa com ferramentas que se } \\
\text { expandem capacidades mentais }\end{array}$ \\
\hline
\end{tabular}


Revista de Educação, Ciência e Tecnologia

\begin{tabular}{|l|l|}
\hline Inteligência Coletiva & $\begin{array}{l}\text { Reunir conhecimento e comparar notas com outros em } \\
\text { direção a um objetivo comum }\end{array}$ \\
\hline Julgamento & $\begin{array}{l}\text { Avaliar a confiabilidade e credibilidade de diferentes } \\
\text { informações fontes }\end{array}$ \\
\hline Navegação transmídia & $\begin{array}{l}\text { Acompanhar o fluxo de histórias e informações através } \\
\text { de múltiplas modalidades }\end{array}$ \\
\hline Networking & Procurar, sintetizar e disseminar informações \\
\hline Negociação & $\begin{array}{l}\text { Viajar através de diversas comunidades, discernindo e } \\
\text { respeitando múltiplas perspectivas, e agarrando e } \\
\text { seguindo normas alternativas }\end{array}$ \\
\hline
\end{tabular}

Fonte: Adaptado pelas autoras - JENKINS et al. (2006) - tradução nossa

Percebe-se que um contexto de cultura digital, de convergência midiática, favorece uma comunicação que ultrapassa leitura e escrita. Ela acomoda linguagens múltiplas, convergentes e compartilhadas em inúmeras redes/plataformas digitais, onde todos podem interagir/atuar/interferir/comunicar/assistir, rompendo um modelo de comunicação massiva e articulando uma teia muito mais complexa e dinâmica de comunicação, que democratiza o acesso à informação, potencializa a produção e promove a autoria e o protagonismo:

\begin{abstract}
A convergência não ocorre por meio de aparelhos, por mais sofisticados que venham a ser. A convergência ocorre dentro dos cérebros de consumidores individuais e em suas interações sociais com outros. Cada um de nós constrói a própria mitologia pessoal, a partir de pedaços e fragmentos de informações extraídos do fluxo midiático e transformados em recursos através dos quais compreendemos nossa vida cotidiana. Por haver mais informações sobre determinado assunto do que alguém possa guardar na cabeça, há um incentivo extra para que conversemos entre nós sobre a mídia que consumimos (JENKINS, 2009, p. 31)
\end{abstract}

O quanto a Educação vislumbra a potência desse contexto? Freire (1987, p. 178) trata acerca da potência de "um momento altamente pedagógico, em que a liderança e o povo fazem juntos o aprendizado da autoridade e da liberdade, verdadeiras que ambos, como um só corpo, buscam instaurar, com a transformação da realidade que os mediatiza". Para a escola, o cenário é de oportunidade de fortalecimento de propostas, onde a tecnologia possibilite o diálogo e ofereça a seus interlocutores novos espaços no fluxo da informação e outros arranjos na construção do conhecimento.

Que mundo a ser explorado pela escola, que mundo a ser construído pela escola, quanta potência pedagógica pode haver em um deslizar de dedos, na tela de um dispositivo móvel? Rojo (2013, p.37) problematiza esta questão: "Posso dizer que nem as tecnologias digitais, nem os novos multiletramentos da cultura digital efetivamente chegaram ainda às práticas escolares, que continuam aferradas ao impresso e suas práticas."

Em muitos aspectos relacionados ao desenvolvimento de um trabalho assistido pela utilização de tecnologias digitais e móveis, a educação é impedida de avançar. A formação dos professores, por exemplo, figura nesta conjuntura ao voltar-se para modelos alinhados a práticas pouco flexíveis, conservadoras na metodologia e no uso de inovações tecnológicas. Brasilino et al. (2018) apontam que "a formação inicial de professores não apresenta suficiente foco no letramento digital, entendido como a habilidade necessária para integrar as TIC na prática pedagógica." 
Ao analisarem a formação docente e letramento digital, Brasilino et al. (2018) mostram que a presença das TIC na formação inicial denota uma "abordagem técnica e não crítica", muito mais orientada para o planejamento das aulas e não para o desenvolvimento de propostas voltadas aos alunos. Apontam também que o oferecimento de uma disciplina sobre o uso das TIC no currículo de formação docente não é tão relevante quanto a integração das TIC a todo o processo de formação.

Pensar sobre a estrutura dos espaços escolares também pode evidenciar relevância nesta discussão. Estudos que apresentam considerações a partir da escuta dos professores que atuam nas escolas corroboram com uma reflexão acerca da importância de um olhar direcionado ao trabalho desenvolvido em espaços que apresentam entraves como equipamentos obsoletos, sucateados (ou a inexistência deles no espaço escolar), isolamento da rede (escolas que ainda não estão conectadas à internet) ou acesso restrito à rede (escolas com acesso à internet, mas sem disponibilização do sinal Wi-fi).

Observa-se que as dificuldades para o uso de tecnologias em atividades pedagógicas entre professores de escolas particulares foram muito semelhantes às citadas por aqueles de escolas públicas, como o número insuficiente de computadores por aluno, atualizados e conectados à Internet; a baixa velocidade de conexão; e a ausência de curso específico para o uso de computador e Internet nas aulas. (COMITÊ GESTOR DA INTERNET NO BRASIL, 2018, p.135)

A necessidade de acompanhar a sociedade, de ser permeável às suas práticas e de contribuir com essas práticas desde sempre se configura um dos desafios para a Educação. $\mathrm{O}$ contexto atual atenua o isolamento, derruba os muros, magnifica os limites, indetermina os espaços e contextos de aprendizagem. O mundo é a sala de aula! Todos os espaços são potencialmente de aprendizagem e estimulam a autoria e o compartilhamento. Somos multivíduos desta metrópole comunicacional, com possibilidade dialógica:

\begin{abstract}
Uma pessoa pode se auto-representar de uma maneira muito mais livre e descentrada do que antes. Agora um jornalista, um pesquisador, um antropólogo, não tem mais a autoridade de representar o outro. Ele tem uma possibilidade dialógica, mas o outro se auto-representa, se auto-narra, se auto-compõe. Esse tipo de auto-representação é parte fundamental do multivíduo da metrópole comunicacional. (CANEVACCI, 2009)
\end{abstract}

O processo de aprendizagem, que anteriormente possibilitaria um trabalho que se referenciasse, por exemplo, em um programa produzido em contexto não convergente com a cultura local, por um meio de comunicação massiva e generalista, mais distanciando o conteúdo do que aproximando-o da realidade dos sujeitos, hoje pode se transformar em processo de produção deste conteúdo, de reflexão sobre ele, das possíveis formas de comunicá-lo e não simplesmente de assisti-lo/recebê-lo bancariamente. Além disso, possibilita processos de construção e produção coletivos e colaborativos:

Nenhum de nós pode saber tudo; cada um de nós sabe alguma coisa; e podemos juntar as peças, se associarmos nossos recursos e unirmos nossas habilidades. A inteligência coletiva pode ser vista como uma fonte alternativa de poder midiático. Estamos 
aprendendo a usar esse poder em nossas interações diárias dentro da cultura da convergência. (JENKINS, 2009, p.30)

Nessa realidade múltipla, multifacetada, fluida, móvel, escancarada a todo tipo de informação, é necessário discernimento, capacidade de leitura, de interpretação da informação, de filtro, de seletividade, de comunicação ética. Essa é a hora da escola, estando em privilegiado espaço de formação de autores e comunicadores, mobilizadores de ações cidadãs de defesa e compartilhamento do conhecimento, da verdade e de valores sociais éticos, agora também em contexto digital. Aliás, a escola se configura como um dos espaços formais que podem conduzir os sujeitos à cidadania digital.

\section{Do caderno para o mundo: visibilidade para o conhecimento científico escolar em contexto de cultura digital}

Argumentos a respeito da importância de propostas de educação em contexto de cultura digital, que desenvolvam voz, autoria, cultura colaborativa já foram apresentados. Principalmente quando a abordagem está vinculada à aprendizagem em ciências e à linguagem científica, a relação entre ciência/academia e comunidade/senso comum é notadamente impessoal, distante. O vocabulário científico é de difícil entendimento à população, restrito a um recorte dito "intelectual" da população em geral, fluentes na linguagem científica.

De acordo com Krasilchik e Marandino (2007), o conhecimento científico escolar não se limita à adaptação da linguagem para a produção de novos saberes. Destacam também que, no ensino formal, o conhecimento ainda está organizado de tal forma que é fragmentado em disciplinas isoladas, o que impede uma articulação relacionada com a vida. Esse formato foi historicamente se alterando, conforme determinavam os fatores políticos e econômicos vigentes em cada reforma de ensino de ciências. Essa sucessão de projetos foi incorporando elementos que, de certa maneira, promoveram a conexão entre o contexto cultural e as disciplinas tradicionais, como os temas transversais, por exemplo. O ensino de Ciências evoluiu para uma visão interdisciplinar ao considerar o contexto da pesquisa científica e suas consequências sociais, políticas e culturais. Esta amplificação nas relações possibilitou o surgimento de um movimento pedagógico denominado Ciência-tecnologia e sociedade (CTS), que leva em conta a importância atual da ciência na tecnologia e envolve uma "visão interdisciplinar que desconsidera rígidas fronteiras dividindo campos de conhecimento" (KRASILCHIK; MARANDINO, 2007, p.4). As autoras também definem as tendências essenciais relacionadas ao conhecimento básico de ciência:

Cursos e programas que enfatizam a memorização de vocabulário são os mais presentes e dão aos alunos ideias distorcidas da ciência como um conjunto de nomes e definições impedindo que vejam as interações da ciência, tecnologia e sociedade. Embora necessário, adquirir vocabulário básico não é o bastante. É necessário levar o estudante a buscar lógica e racionalmente, mas também criticamente, os dados empíricos que devem ser de domínio público. A formação do aprendiz deve levá-lo a compreender que o conhecimento científico é cumulativo e historicamente arquitetado, tendo sempre caráter tentativo. Comporta, por isso, rupturas e está implicado nas relações sociais, políticas, econômicas e ideológicas das sociedades onde é produzido. (KRASILCHIK; MARANDINO, 2007, p.15) 
Estas mudanças demandam posicionamento em relação à construção do currículo voltado ao ensino de ciências e exigem que o professor lance mão de metodologias e instrumentos que atendam a estas demandas:

\begin{abstract}
Que ciência e que tecnologia deve ser compreendida pela população? Para que? Como? Quem deve tomar essas decisões? Os cientistas? Os divulgadores? Os professores? A família? O conjunto dos cidadãos? Decidir qual a informação básica para viver no mundo moderno é hoje uma obrigação para os que acreditam que a educação é um poderoso instrumento para combater e impedir a exclusão e dar aos educandos de todas as idades possibilidades de superação dos obstáculos que tendem a mantê-los analfabetos. O presente estado de coisas somente será modificado com uma corajosa ação de renovação curricular incluindo programas e metodologias adequadas às atuais questões sociais. A expressão "ciência para todos" que para muitos resume esta postura, além de levar em conta experiências prévias, exige também seleção de tópicos que tenham significado para os cidadãos e possam servir de base e orientação para suas decisões pessoais e sociais, principalmente as que envolvem questões éticas. (KRASILCHIK; MARANDINO, 2007, p.10)
\end{abstract}

Ao tratar sobre a questão da popularização do conhecimento científico, a autora também propõe uma reflexão sobre a socialização do conhecimento, indicando que essa prática está conectada a questões culturais com o propósito de utilizar linguagens que apresentem os saberes em formatos mais acessíveis aos indivíduos, ressaltando que os termos "diálogo" e "mediação" são determinantes no processo de divulgação da ciência: "Trata-se assim de um processo de diálogo entre diferentes elementos da cultura - a científica, o senso comum, os conhecimentos dos variados grupos sociais" (p.24).

Um estudante fluente nessas diversas e convergentes linguagens saberá construir uma narrativa para visibilizar o conhecimento construído. Qual o espaço que a visibilidade dessas construções dos estudantes ocupa na ação escolar, no projeto político pedagógico, em seu planejamento? A Base Nacional Comum Curricular - BNCC (BRASIL, 2017) já aponta algumas questões dentro desta perspectiva de ensino em contexto de cultura digital:

\begin{abstract}
Será dada ênfase especial a procedimentos de busca, tratamento e análise de dados e informações e a formas variadas de registro e socialização de estudos e pesquisas, que envolvem não só os gêneros já consagrados, como apresentação oral e ensaio escolar, como também outros gêneros da cultura digital - relatos multimidiáticos, verbetes de enciclopédias colaborativas, vídeos-minuto etc. Trata-se de fomentar uma formação que possibilite o trato crítico e criterioso das informações e dados. (BRASIL, 2017, p.138)
\end{abstract}

A escola, ao se apropriar de outras formas de apresentação de suas pesquisas, de suas produções, estende-se além do texto convencional, resumos e ensaios. A linguagem digital, imagética, audiovisual disponibilizada em wikis, blogs, flogs, redes sociais, plataformas de conteúdo volátil e de todo tipo de conteúdo digital, tão sedutor e abundante, pode ser utilizada como fonte ou objeto de estudo para a construção do conhecimento científico escolar, mas também apresenta um potente recurso visibilizador da escola e suas produções, de disseminação de suas importantes e relevantes contribuições para a comunidade, que permite à escola mostrar (e em alguns casos até resgatar) sua importância próximo ao senso comum do cotidiano, da vida. 


\title{
4 Considerações Finais
}

Quando inovar em educação significa abandonar um modelo padronizado de escola, que tipo de fazer pedagógico mais se afinaria a essa conjuntura do que subverter um modelo tradicional, em favor da formação de sujeitos que atuem ativa e colaborativamente em um processo de construção de saberes relações com o mundo? O que pode ser mais atual do que uma escola que possibilite o atendimento às demandas dos alunos e suas comunidades, desenvolvendo um trabalho que oportunize o desenvolvimento de um sujeito mais atuante em seu aprendizado e que o permita ressignificar, questionar e modificar a sua realidade e o mundo? Necessário identificar as diversas maneiras de como se ensina e aprende, com potencial de engajar alunos e comunidade na educação (BOLL et al, 2018) visto que, nas palavras das autoras: "[...] hoje a mobilidade oferecida pela Cultura Digital garante não só outras formas de sociabilidades na esfera da comunicação e da mídia, mas também outras formas de ensinar e aprender na esfera escolar" (BOLL et al, 2018, p.66).

Neste momento, a Cultura Digital, que dinamiza as relações comunicativas, estabelece novos processos sociais, amplifica as formas de acesso e disseminação da informação, oportuniza à escola, muito mais do que uma reflexão sobre o sujeito que estamos formando, ações efetivas para essa formação.

Em um cenário comunicacional onde quase todo tipo de informação pode circular, há de se pensar sobre, ao menos, dois aspectos: ou a escola deixa de aproveitar esse cenário, cedendo espaço para outras intenções na disseminação e visibilização da informação, ou ela toma posse de seu papel de formadora de sujeitos mobilizadores, comunicadores e disseminadores do conhecimento, da arte e de suas construções sob uma perspectiva ética e estética de comunicação. Para isso, é importante que a escola se perceba pertencente a esse contexto, atuando nesta condição, como afirmam Boll e Kreutz (2009):

\begin{abstract}
Mas como é possível a escola apropriar-se da Cultura Digital em favor do pedagógico? Assim como uma abelhinha que entra na sala pode gerar um fenômeno que se transforme em foco de interesse de uma turma do Ensino Fundamental, fazendo dela todo o seu projeto de estudo, a CULTURA pulsante do DIGITAL está à espera de ser descoberta pelo educador e até mesmo pelo aluno que, também, ainda não a vê neste contexto. (BOLL; KREUTZ, 2009, p. 16)
\end{abstract}

Muitas tecnologias podem atuar como suporte nesta missão, mas não somente para etapas de construção do conhecimento como a pesquisa na rede, a utilização de softwares/aplicativos de aprendizagens. A questão que aqui se levanta é a da produção, da potência criadora contribuindo com o ensino de ciências e a construção do conhecimento científico em um contexto de cultura digital. Ao considerar o uso da tecnologia no processo comunicacional do conhecimento científico escolar, a escola lança mão de infinitas possibilidades de tornar visível, publicizando as construções promovidas durante o processo de aprendizagem.

Considerando a mídia como um mecanismo que potencializa a transmissão de informação (e aí estariam incluídos os meios de comunicação social: TV, rádio, cinema, mídia impressa, entre outros) é inconcebível que não se considere seu uso na escola. Ao mesmo tempo, é preciso considerar que esses recursos necessitam de mediação e de orientação para que não sejam simplesmente apresentados como algo a ser visto/ouvido/lido sem que se leve em conta suas possibilidades na promoção do protagonismo dos sujeitos. (RAMOS; LEMOS, 2018, p.148) 
Uma das possibilidades que, em contexto de cultura digital, se apresenta potente no espaço escolar é a produção audiovisual. Campos et al. (2018) discorrem a respeito da importância de um trabalho que vá além da utilização do recurso audiovisual de forma ilustrativa, mas que considere as potencialidades desta linguagem:

É significativo, portanto, sistematizar e viabilizar a construção coletiva e colaborativa de uma proposta para a educação audiovisual que assuma seu papel para a ação pedagógica desenvolvida entre professor, aluno e comunidade, com apoio da tecnologia. Esse processo amplia o repertório de gêneros, estilos e narrativas que colocam o cinema como forma de conhecimento e experiência de mundo. (CAMPOS et al., 2018 p.213)

O cenário ultrapassa a possibilidade de uma ação isolada. Ele é múltiplo, permite ir além e o panorama é convergente. Produção de vídeos, podcasts, elaboração de arte e conteúdo digital, utilização de plataformas diversas na hospedagem e publicação são ações potentes ao fazer pedagógico na escola e para além dela, tornando o conhecimento científico mais acessível e aproximando comunidade e espaços formais de educação. $\mathrm{O}$ texto não é o único suporte. $\mathrm{O}$ professor não é o único receptor das construções desse sujeito. As descobertas dos estudantes não precisam ficar restritas aos murais da escola, à feira de iniciação científica e ao público deste evento. O mundo é a sala de aula e a escola encontra, neste contexto, a potência e a exigência de um trabalho orientado para a exploração de um universo comunicacional. Ao ingressar nessa metrópole comunicacional, ela reduz o isolamento, levando à comunidade suas construções, contribuindo para uma sociedade cidadã digital, mostrando a potência de um trabalho embebido em cibercultura, ciência e produção do conhecimento científico escolar.

\section{Referências}

BOLL, Cíntia Inês; KREUTZ, José Ricardo. Caderno Cultura Digital. Brasília: PDE MEC, 2009. 51 p. (Cadernos Pedagógicos - Mais Educação). Disponível em: $<$ http://portal.mec.gov.br/index.php?option=com_docman\&view=download\&alias=12330culturadigital-pdf\&Itemid=30192>. Acesso em: 08 mar. 2019.

BOLL, Cíntia Inês; CORBELLINI, Silvana; GALAFASSI, Fabiane. O Wikilivros e as mídias móveis: um exemplo de como as diretrizes curriculares nacionais para a educação básica podem ser reutilizadas, revisadas, remixadas e redistribuídas entre professores, gestores appers. In: Mariângela Bairros; Patrícia Marchand. (org.). Coordenador pedagógico: concepções e práticas. 1ed.Porto Alegre: Tomo Editorial, 2018, v. 1, p. 63-80.

BRASIL. Ministério da Educação. Base Nacional Comum Curricular. Brasília, 2017. 600 p. Disponível em: <http://basenacionalcomum.mec.gov.br/images/BNCC_EI_EF_110518_versaofinal_site.pdf> . Acesso em: 26 maio 2019.

BRASILINO, Aline de Mendonça et al. Formação docente e letramento digital: uma análise de correlação na base da pesquisa TIC educação. In: Centro Regional de Estudos para o Desenvolvimento da Sociedade da Informação (Cetic.br). Pesquisa sobre o uso das 
Tecnologias de Informação e Comunicação nas escolas brasileiras - TIC Educação 2017. São Paulo: Nic.br / Cetic.br, 2018. p. 35-42. Disponível em:

<https://cetic.br/media/docs/publicacoes/2/tic_edu_2017_livro_eletronico.pdf >.Acesso em: 25 jul. 2019.

CAMPOS, Lucas Miranda; LEMOS, Cristina Domingues; RAMOS, Luciana Domingues. Núcleo de Educação Audiovisual - Uma proposta de política pública de fomento à produção audiovisual na Rede Pública Municipal de São Leopoldo. In SANTOS, Maria Angélica; LAZZARETI, Angelene; COSTA, Juliana Vieira (Orgs). Escritos de alfabetização audiovisual: luz na docência. Porto Alegre: Cinemateca Capitólio, 2018, p.205-213

CANEVACCI, Massimo. Comunicação entre corpos e metrópoles. Signos do Consumo, São Paulo, v. 1, n. 1, p.8-20, 16 jun. 2009. Disponível em:

<http://www.revistas.usp.br/signosdoconsumo/article/view/42762/46416>. Acesso em: 07 jun. 2019.

COMITÊ GESTOR DA INTERNET NO BRASIL. Pesquisa sobre o uso das Tecnologias de Informação e Comunicação nas escolas brasileiras - TIC Educação 2017. São Paulo: Nic.br / Cetic.br, 2018. 435 p. Disponível em:

<https://cetic.br/media/docs/publicacoes/2/tic_edu_2017_livro_eletronico.pdf>. Acesso em: 25 jul. 2019.

FRAGA, Dinorá. Linguagem, tecnociência e tecnocultura. In: FRAGA, Dinorá; AXT, Margarete. (org.). Políticas do virtual: inscrições em linguagem, cognição e educação. São Leopoldo: Unisinos, 2012, p. 23-34.

FREIRE, Paulo. Educação como prática da liberdade. Rio de Janeiro: Paz e Terra, 1967. $149 \mathrm{p}$.

FREIRE, Paulo. Pedagogia do Oprimido. 27. ed. Rio de Janeiro: Paz e Terra, 1987.

JENKINS, Henry et al. Confronting the Challenges of Participatory Culture: Media Education for the 21st Century. Chicago: Macarthur Foundation, 2006. Disponível em: $<$ https://www.macfound.org/media/article_pdfs/JENKINS_WHITE_PAPER.PDF>. Acesso em: 24 maio 2019.

JENKINS, Henry. Cultura da Convergência. 2. ed. São Paulo: Aleph, 2009.

KRASILCHIK, Myriam; MARANDINO, Martha. Versão não publicada do livro: Ensino de Ciências e Cidadania. São Paulo: E-disciplinas Usp, 2007. 53 p. Disponível em: <https://edisciplinas.usp.br/mod/resource/view.php?id=516684>. Acesso em: 27 jul. 2019.

LÉVY, Pierre. Cibercultura. Rio de Janeiro: 34, 1999. 264 p.

MORAN, José. Metodologias ativas para uma aprendizagem mais profunda. 2013. Disponível em: <http://www2.eca.usp.br/moran/wpcontent/uploads/2013/12/metodologias_moran1.pdf >. Acesso em: 01 maio 2019. 
RAMOS, Luciana Domingues; LEMOS, Cristina Domingues. Mídia e produção audiovisual: suporte para o ciclo de alfabetização. In CANDIDO, Eliane; PEREIRA, Josias (org.). São Léo em Cine: a escola construindo sonhos. São Leopoldo-RS: Editora ERD Filmes, 2018, p.146-131

ROJO, Roxane Helena Rodrigues. Desafios dos (multi)letramentos nas nuvens. Na Ponta do Lápis, São Paulo, v. 1, p.36-37, 22 ago. 2013. Disponível em:

$<$ https://www.escrevendoofuturo.org.br/EscrevendoFuturo/arquivos/968/NPL22.pdf>. Acesso em: 16 jun. 2019.

Recebido em agosto de 2019.

Aprovado em setembro de 2019. 